\title{
A new diagnostic tool for masked hypertension: impaired sleep quality
}

\author{
Fatma Erdem¹, Ugur Cakır², Osman Yıldırım², Aytekin Alcelik ${ }^{3}$, Ibrahim Donmez ${ }^{1}$, \\ Taha Can Tuman², Sabri Onur Caglar ${ }^{1}$, Alim Erdem¹, Mehmet YazıcI ${ }^{1}$
}

\author{
${ }^{1}$ Department of Cardiology, Faculty of Medicine, Abant Izzet Baysal University, Bolu, \\ Turkey \\ 2Department of Psychiatry, Faculty of Medicine, Abant Izzet Baysal University, Bolu, \\ Turkey \\ ${ }^{3}$ Department of Internal Medicine, Faculty of Medicine, Abant Izzet Baysal University, \\ Bolu, Turkey
}

Submitted: 15 December 2015

Accepted: 25 January 2016

Arch Med Sci 2016; 12, 6: 1207-1213

DOI: 10.5114/aoms.2015.51177

Copyright $\odot 2016$ Termedia \& Banach

\section{Abstract}

Introduction: The aim of this study was to evaluate the relationship between masked hypertension and impaired sleep quality. Additionally, we evaluated the diagnostic role and prevalence of poor sleep quality among patients with newly diagnosed masked hypertension.

Material and methods: A total of 112 individuals, 72 patients with newly diagnosed masked hypertension and 40 normotensive healthy volunteers, were included in this study. All patients underwent evaluation comprising 12-lead electrocardiography, transthoracic echocardiography, 24-hour Holter ECG, and basic laboratory tests. Additionally, all participants completed questionnaires, including the Pittsburgh Sleep Quality Index (PSQI).

Results: The total PSQI score was significantly higher in the masked hypertension group than in the normotensive healthy volunteers $(4.13 \pm 2.43$ vs. $2.33 \pm 1.67, p<0.001)$. A PSQI score $>5$ was found in $45.8 \%(n=33)$ of patients in the masked hypertension group and $15 \%(n=6)$ of patients in the normotensive group $(p<0.001)$. The non-dipper pattern was found in $17.5 \%$ of the healthy volunteer group and $59.94 \%(n=41)$ of the masked hypertension group $(p<0.001)$. When we compared the dipping pattern of the masked hypertension groups, there was a significant difference in PSQI score between the dipper and non-dipper groups ( $4.87 \pm 3.21$ vs. $3.58 \pm 2.33$, $p<0.001)$. Multiple logistic regression analyses showed that masked hypertension, LV mass, and LV mass index score were independent predictors of poor PSQI.

Conclusions: This study demonstrates impaired sleep quality in subjects with masked hypertension, particularly those with a non-dipper pattern. Additionally, this study indicates that impaired sleep quality may help diagnose masked hypertension, particularly in the non-dipper group.

Key words: masked hypertension, sleep quality, sleep disturbances.

\section{Introduction}

Sleep is an important physiologic process, and sleep disturbances have been shown to negatively influence many aspects of quality of life, including general health, physical functions, cognitive functions, daily activities, and work performance [1]. Previous studies have determined that

\author{
Corresponding author: \\ Alim Erdem MD \\ Department of Cardiology \\ Faculty of Medicine \\ Abant Izzet Baysal University \\ 14280 Bolu, Turkey \\ Phone: +90 5317741266 \\ E-mail: \\ cardiology14@gmail.com
}


impaired sleep quality is common in hypertensive patients and may be associated with lower health-related quality of life $[2,3]$. Moreover, poor sleep quality has been significantly associated with resistance to treatment in hypertensive patients [2].

Masked hypertension (MHT) is clinically defined as normal blood pressure (BP) levels during clinic monitoring with an average systolic $\mathrm{BP} \geq 135 \mathrm{~mm}$ $\mathrm{Hg}$ and diastolic BP $\geq 85 \mathrm{~mm} \mathrm{Hg}$ following $24 \mathrm{~h}$ of ambulatory monitoring [4]. The clinical importance of MHT has been underscored recently by reports of higher cardiovascular mortality and morbidity, as well as higher prevalence of target organ damage, in patients with MHT than in patients with normal clinic and ambulatory BP readings [5, 6]. Moreover, obstructive sleep apnea syndrome has been independently associated with an increased rate of MHT [7, 8]. Diagnosing MHT can be difficult in the outpatient clinics, and the diagnostic period for a first appointment with an outpatient clinic can be long. The detailed history, physical examination, and electrocardiograph are of limited value in the diagnosis of MHT. However, sleep quality in patients with MHT has not yet been reported in the literature.

We hypothesized that there is a relationship between MHT and impaired sleep quality. Thus, in this study, we evaluated the diagnostic role and prevalence of poor sleep quality among patients with newly diagnosed masked hypertension.

\section{Material and methods}

\section{Subjects and study protocol}

We selected 72 newly diagnosed MHT patients and 40 normotensive healthy volunteers well balanced for gender, age, and body mass index (BMI). All patients underwent evaluation comprising 12-lead electrocardiography (ECG), transthoracic echocardiography, 24-hour Holter ECG, and basic laboratory tests. Height and weight were measured for calculation of the $\mathrm{BMI}(\mathrm{BMI}=$ weight $(\mathrm{kg}) /$ height $\left.^{2}\left(\mathrm{~m}^{2}\right)\right)$. The inclusion criteria for the study group were: age $\geq 18$ years, patient's informed consent granted, absence of any acute disease, no administration of B-group vitamins or vitamin preparations within 6 months before the study and normotensive in physical treatment. Patients with any of the following features were excluded from participation: night-time workers, patients with valvular heart disease or chronic obstructive pulmonary disease (identified by a chest physician), patients with a history of sleep apnea/ hypopnea, patients with recent acute coronary syndrome (within the last 2 months), significant systemic disease, history of any psychiatric disease including primary insomnia, symptomatic cerebrovascular disease (including previous transient ischemic attack within 6 months), obstructive coronary artery disease, history of congestive heart failure, hepatic failure, hypertension above study criteria as well as suspicion of secondary hypertension forms, with serum creatinine $>1.4 \mathrm{mg} / \mathrm{dl}$, patients with a history of diabetes mellitus (or fasting blood glucose > $125 \mathrm{mg} / \mathrm{dl}$ ), pregnant women, patients with hypo-hyperthyroidism, patients with a BMI > $25 \mathrm{~kg} / \mathrm{m}^{2}$, and patients who wished to consume alcohol during the study period. Patients using medications which are known for their negative impact on sleep quality, diuretics, $\beta$ blocking agents which disturb sleep quality because of increased urine output, major psychotropic agents, oral steroids, daily nonsteroidal antiinflammatory drugs, or high-dose acetylsalicylic acid were not included in the study.

\section{Office blood pressure measurement}

Clinical systolic and diastolic BP recordings were performed according to the standard technique using a mercury sphygmomanometer. Blood pressure was measured in the sitting position on the right arm. Measurements were repeated three times, and the average value was used as the BP.

\section{Ambulatory blood pressure monitoring}

Twenty-four-hour BP was measured by the cuff-oscillometric method using the ambulatory BP monitor Scanlight III version B (Medset Electronics, Hamburg, Germany). Blood pressure was measured every 30 min from 6 a.m. to midnight and every $60 \mathrm{~min}$ from midnight to $6 \mathrm{a} . \mathrm{m}$. the following day. Daytime and nighttime BPs were obtained as the average values in the awake period between 6 a.m. and midnight and in the sleeping period between midnight and 6 a.m. The average 24-hour systolic and diastolic BPs were evaluated. All subjects were divided into two groups, dipper and non-dipper, according to the percentage of reduction in their nighttime BPs. Subjects were defined as dippers when nighttime systolic and diastolic BPs decreased $\geq 10 \%$ and as non-dippers when nighttime BP decreased < $10 \%$. Within patients having < 140/90 mm Hg office BP, those having $\geq 135 / 85$ average BP values in the day period of 24-hour ambulatory BP monitoring records were evaluated as masked hypertensive.

\section{Echocardiography}

An experienced echocardiographer performed the echocardiographic studies to exclude structural heart disease. All patients were evaluated by transthoracic M-mode and two-dimensional pulsed wave, continuous wave, color flow, and tissue Doppler imaging modalities. All examinations were performed with the GE-Vivid-3 system 
(GE Vingmed, Horten, Norway) with a 2-4 MHz transducer at a depth of $16 \mathrm{~cm}$. Two-dimensional Doppler echocardiographic examinations and M-mode measurements were taken according to the recommendations of the American Society of Echocardiography [9].

\section{Measurement of sleep quality}

The Pittsburgh Sleep Quality Index (PSQI) is a standardized questionnaire to retrospectively document subjective sleep quality during the past 4 weeks [10]. This instrument is composed of 10 questions, distributed in seven components, as follows: Sleep duration, Subject sleep quality, Sleep latency, Habitual sleep efficiency, Sleep disorders, Use of medication to sleep, and Daytime somnolence and disorders. Each category can score up to three points. The instrument total score varies from 0 to 21 points; the higher the score is, the lower is the sleep quality. A score $>5$ points indicates low sleep quality.

\section{Statistical analysis}

Descriptive statistics were performed and all data were expressed as mean \pm SD and percentage ratio. The normality of data was tested using the Kolmogorov-Smirnov test. Parameter differences between the 2 groups were evaluated using the one-way ANOVA test for normally distributed variables and the Kruskal-Wallis test for non-normally distributed variables. For post hoc analysis of normally distributed variables Tukey's $b$ test was used. For post hoc analysis of non-normally distributed variable origin, the Bonferroni-corrected Mann-Whitney U-test was used. For comparison of categorical variables, the $\chi^{2}$ test or Fisher's exact test was used as appropriate. Pearson's and Spearman's correlation coefficients were used to test the relationship between the systolic/diastolic arterial tension and PSQI scores. Multivariate logistic regression was conducted to identify statistically significant variables associated with PSQI scores. The results were considered significant when $p$ was less than 0.05 . We used the software package SPSS 20.0 (SPSS Inc., Chicago, IL, USA) for statistical analysis.

\section{Results}

Initially 230 patients were included. One hundred twelve patients met the inclusion criteria and 118 patients were excluded. A total of 112 individuals were included in the present study. Recruitment, exclusion, and subsequent grouping of all patients are shown in the flowchart (Figure 1). Two groups of patients were compared: the MHT group ( $n=72)$ and the control group ( $n=40$; normotensive healthy volunteers). Both groups were diagnosed during the study period using 24-hour ambulatory BP measurement.

Baseline clinical and demographic characteristics of the patients were similar in both groups (Table I). Comparison of clinical variables between groups showed that only systolic blood pressure, diastolic BP and dipper/non-dipper value levels were significantly different between groups $(p<0.05)$. The non-dipper pattern was found in $17.5 \%$ of the healthy volunteer group and in 59.94\% $(n=41)$ of the MHT group $(p<0.001)$.

The comparison of echocardiographic parameters: left ventricular hypertrophy (LVH), including interventricular septum thickness (IVST), ejection fraction (EF), E/A ratio, and presence of left ventricular diastolic dysfunction (LVDD); IVST, the presence of LVH and LV posterior wall thickness, right wall thickness, LV mass, and LV mass index were significantly higher, and E/A ratio was significantly lower in the MHT group ( $p<0.001$ for all comparisons). The total comparison of electrocardiographic and echocardiography findings between the groups is presented in Table II.

The PSQI total score was significantly higher in the MHT group than in normotensive healthy volunteers ( $4.13 \pm 2.43$ vs. $2.33 \pm 1.67, p<0.001$, Figure 2). Poor sleep quality (PSQI $>5$ ) was found in $45.8 \%(n=33)$ of the MHT group and $15 \%$ $(n=6)$ of the healthy group $(p<0.001)$. When we compare MHT groups by dipping pattern, there was a significant difference in PSQI score between dipper and non-dipper groups (4.87 \pm 3.21 vs. 3.58 $\pm 2.33, p<0.001$ ). In addition, there was a significant positive correlation between PSQI score and systolic TA values $(r=0.563, p<0.001)$.

Multiple logistic regression analyses showed that MHT (odds ratio $(\mathrm{OR})=6.79 ; 95 \%$ confidence interval $(\mathrm{Cl}): 1.24-21.3 ; p<0.001)$, LV mass $(\mathrm{OR}=2.53 ; 95 \% \mathrm{Cl}: 1.46-6.72 ; p<0.001)$, and LV mass index score $(\mathrm{OR}=2.19 ; 95 \% \mathrm{Cl}: 1.42-5.76$; $p<0.001)$ were factors that could independently predict poor PSQI (PSQI score $>5$ points indicates low sleep quality).

\begin{tabular}{|c|c|c|c|}
\hline $\begin{array}{c}\text { Initial, } 230 \text { patients included } \\
\text { the study, who admitted the } \\
\text { outpatient clinic }\end{array}$ & $\begin{array}{c}\text { *105 patients excluded with } \\
\text { stated exclusion criteria }\end{array}$ & $\begin{array}{c}\text { Thirteen patients excluded } \\
\text { beacuase of uncompleted } \\
\text { datas }\end{array}$ & $\begin{array}{c}\text { Masked hypertension group } \\
(n=72)\end{array}$ \\
\cline { 3 - 4 } & $\begin{array}{c}\text { Normotensive healthy } \\
\text { volunteers group ( } n=40)\end{array}$ \\
\hline
\end{tabular}

Figure 1. Recruitment, exclusion, and subsequent grouping of all patients 
Table I. Comparative demographic, clinical, and laboratory parameters in the 2 groups of patients

\begin{tabular}{|c|c|c|c|}
\hline Parameter & MHT $(n=72)$ & Control $(n=40)$ & $P$-value \\
\hline Age [years] $]^{*}$ & $47.4 \pm 11.3$ & $46.1 \pm 10.2$ & $>0.05^{* *}$ \\
\hline Gender (male/female)], $N$ & $42 / 30$ & $26 / 14$ & $>0.05^{* * *+}$ \\
\hline Body mass index $\left[\mathrm{kg} / \mathrm{m}^{2}\right]$ & $26.2 \pm 2.3$ & $25.7 \pm 2.5$ & $>0.05^{* *}$ \\
\hline Waist circumference $[\mathrm{cm}]$ & $87.2 \pm 2.9$ & $86.9 \pm 2.6$ & $>0.05^{* *}$ \\
\hline Smoker/non-smoker, $N$ & $32 / 40$ & $14 / 26$ & $>0.05^{*+*+}$ \\
\hline SBP $[24 h]^{*}$ & $151.2 \pm 23.1$ & $129.1 \pm 11.2$ & $<0.001^{* *}$ \\
\hline $\operatorname{DBP}[24 \mathrm{~h}]^{*}$ & $96.4 \pm 12.2$ & $81.2 \pm 5.6$ & $<0.001^{* *}$ \\
\hline Dipper vs. non-dipper, $N$ & $31 / 41$ & $33 / 7$ & $0.0013^{* * *}$ \\
\hline Metabolic syndrome (present/absent), $N$ & $23 / 49$ & $11 / 29$ & $>0.05^{* * *}$ \\
\hline Fasting blood glucose $[\mathrm{mmol} / \mathrm{l}]$ & $5.11 \pm 0.51$ & $5.01 \pm 0.43$ & $>0.05^{* *}$ \\
\hline Blood urea nitrogen [mmol/l] & $5.31 \pm 0.54$ & $5.06 \pm 0.41$ & $>0.05^{* *}$ \\
\hline Creatinine $[\mu \mathrm{mol} / \mathrm{l}]$ & $79.3 \pm 29.2$ & $75.3 \pm 18.2$ & $>0.05^{* *}$ \\
\hline Hemoglobin $[\mathrm{g} / \mathrm{l}]$ & $143.7 \pm 42.3$ & $140.5 \pm 34.3$ & $>0.05^{* *}$ \\
\hline Sodium $[\mathrm{mmol} / \mathrm{l}]$ & $141.5 \pm 8.21$ & $140.9 \pm 8.33$ & $>0.05^{* *}$ \\
\hline Potassium [mmol/l] & $4.33 \pm 0.35$ & $4.29 \pm 0.23$ & $>0.05^{* *}$ \\
\hline Calcium $[\mathrm{mmol} / \mathrm{l}]$ & $2.33 \pm 0.11$ & $2.41 \pm 0.11$ & $>0.05^{* *}$ \\
\hline Phosphorus [mmol/l] & $1.13 \pm 0.14$ & $1.09 \pm 0.12$ & $>0.05^{* *}$ \\
\hline Total cholesterol [mmol/l] & $5.33 \pm 1.16$ & $5.21 \pm 1.01$ & $>0.05^{* *}$ \\
\hline LDL cholesterol [mmol/l] & $3.19 \pm 0.88$ & $3.06 \pm 0.81$ & $>0.05^{* *}$ \\
\hline HDL cholesterol [mmol/l] & $1.31 \pm 0.36$ & $1.30 \pm 0.29$ & $>0.05^{* *}$ \\
\hline Triglyceride $[\mathrm{mmol} / \mathrm{l}]$ & $1.58 \pm 0.72$ & $1.49 \pm 0.65$ & $>0.05^{* *}$ \\
\hline
\end{tabular}

SBP - systolic blood pressure, DBP - diastolic blood pressure, $L D L$ - low-density lipoprotein, HDL - high-density lipoprotein. ${ }^{*} M e a n \pm$ standard deviation; ${ }^{* *} p$-value is based on one-way ANOVA test; ${ }^{* *} p$-value is based on chi-square test or Fisher's exact test as appropriate.

Table II. Comparison of electrocardiographic and echocardiographic findings between groups

\begin{tabular}{|c|c|c|c|}
\hline Parameter & MHT $(n=72)$ & Control $(n=40)$ & $P$-value \\
\hline LVH (based on ECG), present/absent, $N$ & $34 / 38$ & $3 / 37$ & $<0.001^{\star * *}$ \\
\hline IVST $[\mathrm{mm}]^{*}$ & $1.23 \pm 0.12$ & $0.92 \pm 0.14$ & $<0.001^{\star *}$ \\
\hline PWT $[\mathrm{mm}]^{*}$ & $1.17 \pm 0.10$ & $0.90 \pm 0.11$ & $<0.001^{\star *}$ \\
\hline $\mathrm{RWT}[\mathrm{mm}]^{*}$ & $0.43 \pm 0.06$ & $0.39 \pm 0.6$ & $>0.05^{\star \star}$ \\
\hline LV mass $[\mathrm{g}]$ & $193.23 \pm 12.21$ & $165.42 \pm 10.42$ & $<0.001^{\star *}$ \\
\hline LV mass index ${ }^{\star \star \star \star}$ & $103.57 \pm 7.24$ & $86.73 \pm 8.75$ & $<0.001^{\star *}$ \\
\hline EF (\%) & $59.11 \pm 9.21$ & $61.21 \pm 11.24$ & $>0.05^{\star *}$ \\
\hline LVDD, present/absent, $N$ & $25 / 47$ & $5 / 35$ & $<0.001^{\star * *}$ \\
\hline E/A ratio & $0.91 \pm 0.15$ & $1.21 \pm 1.02$ & $<0.001^{* *}$ \\
\hline
\end{tabular}

LVH - left ventricular hypertrophy, IVST - interventricular septum, EF - ejection fraction, LVDD - left ventricle diastolic dysfunction, $P W T$ - posterior wall thickness, RWT - right ventricular wall thickness. ${ }^{*}$ Mean \pm standard deviation; ${ }^{* *}$-value is based on one-way ANOVA test; ${ }^{* * *} p$-value is based on chi-square test or Fisher's exact test as appropriate. ${ }^{* * * *} L V H$ defined as $L V$ mass index of $>116.0$ in men and $>104.0$ in women. 


\section{Discussion}

This is the first study to evaluate sleep quality using Pittsburgh Sleep Quality Index (PSQI) scores in newly diagnosed MHT patients. Our results indicate significantly greater impairment of sleep quality scores among MHT patients than normotensive subjects. In addition, a non-dipper pattern was significantly more common in MHT patients than in normotensive subjects, and it was correlated with higher PSQI scores. Thus, sleep quality was significantly disturbed in non-dipper MHT patients, and the frequency of poor sleep quality, defined as a PSQI score > 5, was greater in MHT patients, particularly those in the non-dipper group. Additionally, this study indicates that impaired sleep quality may help diagnose MHT, particularly in the non-dipper group.

The importance of MHT was recognized a decade ago in a study by Pickering et al. [11] that introduced the term MHT to describe individuals with normal clinic BP levels ( $<140 / 90 \mathrm{~mm} \mathrm{Hg}$ ) and ambulatory hypertension (awake $\geq 135 / 85 \mathrm{~mm} \mathrm{Hg}$ ). Previous studies showed a clear association between MHT and an increased risk of cardiovascular disease events with increased cardiovascular end-organ damage [12-14]. A meta-analysis of observational studies by Trevisol et al. [15] showed that quality of life and sleep quality in hypertensive patients were worse than in normotensive individuals. Taken together, these results revealed that hypertension and impaired sleep quality are common and may be associated with lower health-related quality of life. Interestingly, an analysis by Palagini et al. [16] showed that impaired sleep quality, short sleep duration, and persistent insomnia were associated with increased BP and increased risk of hypertension, even after controlling for other risk factors. They indicated that alterations in sleep quality might act as a neurobiological and physiologic stressor resulting in increased arterial pressure and hypertension.

This is the first study to examine sleep quality in patients with MHT. We determined that sleep quality was impaired in patients with $\mathrm{MHT}$, which may disrupt appetitive hormone regulation and play a role in the pathogenesis of MHT. Poor sleep quality may be an important factor to consider in the diagnosis and treatment of MHT because evaluating sleeping status and sleep quality may help unmask MHT, enabling physicians to diagnose and appropriately treat MHT more effectively. Additional studies are needed to confirm the high prevalence of impaired quality of sleep in this population and to evaluate the pathophysiologic mechanisms linking MHT and impaired sleep quality.

In addition, our results suggest a close relationship between the non-dipper pattern, MHT, and impaired sleep quality. Erden et al. [17] found that

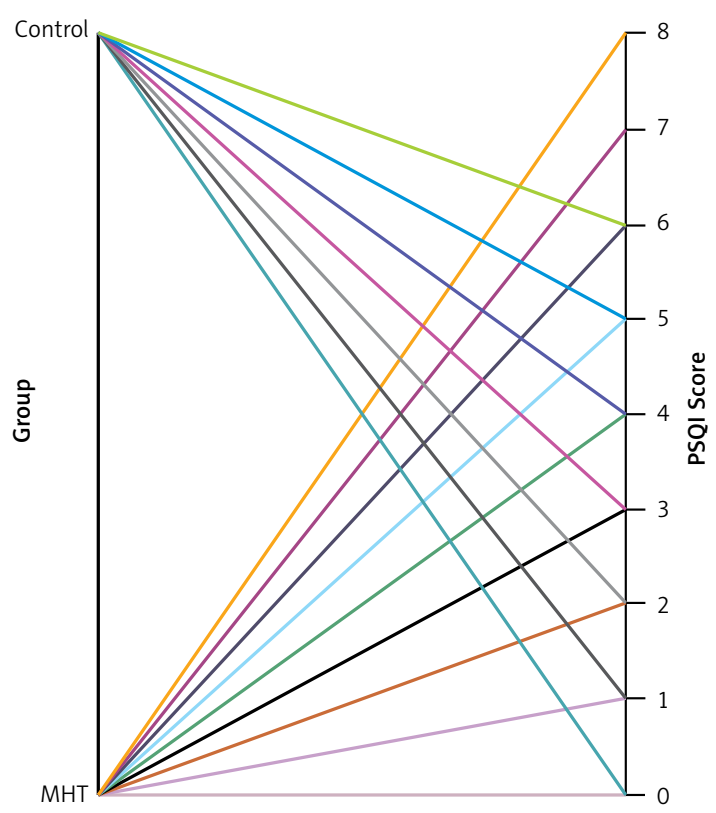

Figure 2. Distribution of PSQI scores in MHT group and normotensive healthy subjects

poor sleep quality was related to a non-dipping pattern and acted as an independent predictor of non-dipping in newly diagnosed stage 1 hypertensive patients. Mansoor [18] used a logistic regression analysis to reveal that nighttime activity was predictive of a non-dipping BP profile and suggested a possible sleep disturbance in non-dipper hypertensive patients. Previous studies have clearly shown an association between a non-dipper pattern and increased cardiovascular disease risk in hypertensive patients [16, 19]. Additionally, previous studies have suggested that normotensive persons with a non-dipper pattern have increased target organ damage [20,21]. The results of this study suggest that MHT patients with a non-dipper pattern and poor sleep quality should be closely monitored by clinicians.

Previous studies clearly showed that MHT predisposes to subclinical organ damage and to increased cardiovascular risk [22-25]. The MHT patients in our study had a significantly higher level of left ventricular hypertrophy (LVH) compared with the control group. Similarly, the Masked Hypertension Study by Shimbo et al. [22] showed that compared to healthy normotensive participants, left ventricular mass index (LVMI) was significantly higher in participants with MHT. Kotsis et al. [23] showed that MHT was accompanied by increased LVMI and carotid intima media thickness, even after adjusting for other risk factors. Interestingly, Mitsnefes et al. [24] suggested that $\mathrm{LVH}$ was more common in children with MHT. Also, in their multivariable analysis, $M H T(O R=4.1)$ was the strongest independent predictor of LVH in children. In addition, we found that left ventricular diastolic function was significantly impaired in MHT 
patients. Oe et al. [25] reported that left ventricular diastolic function was impaired in MHT compared with normotensive subjects independent of changes in left ventricular mass. Based on these results, we suggest that various subclinical organ damage and increased cardiovascular risk are observed in individuals with MHT. Clinicians should focus more on MHT patients for subclinical organ damage and increased cardiovascular risk.

Some limitations of this study should be discussed. The primary limitation of our study was the small sample size and selections of participants. A small sample size has low statistical power and thus may yield false-negative results. Also, PSQI scores are affected by many factors, but we tried to use some exclusion criteria to reduce that limitation. Accordingly, we cannot apply our results to the general population due to the broad exclusion criteria. Furthermore, the other limitation of our study is its cross-sectional design. Thus, our results cannot be extended to the general population. However, we believe that our findings provide a valuable contribution to the study of masked hypertension and sleep quality. Future prospective much larger multicenter studies are required to confirm our results.

In conclusion, this study demonstrates impaired sleep quality in subjects with $\mathrm{MHT}$, particularly those with a non-dipper pattern. Additionally, this study indicates that impaired sleep quality may help diagnose MHT, particularly in the non-dipper group. Our findings will serve as the basis for future studies of the non-dipper BP pattern, sleep quality, and MHT. We should also emphasize that in this study, in contrast to previous reports, the subjects with MHT were evaluated in terms of sleep quality.

\section{Conflict of interest}

The authors declare no conflict of interest.

\section{References}

1. Dinges DF, Pack F, Williams K, et al. Cumulative sleepiness, mood disturbance, and psychomotor vigilance performance decrements during a week of sleep restricted to 4-5 hours per night. Sleep 1997; 20: 267-77.

2. Bruno RM, Palagini L, Gemignani A, et al. Poor sleep quality and resistant hypertension. Sleep Med 2013; 14: 1157-63.

3. Parish JM. Sleep-related problems in common medical conditions. Chest 2009; 135: 563-72.

4. Angeli F, Reboldi G, Verdecchia P. Masked hypertension: evaluation, prognosis, and treatment. Am J Hypertens 2010; 23: 941-8

5. Yoon HJ, Ahn Y, Park JB, et al.; Korean Hypertension Research Network. Are metabolic risk factors and target organ damage more frequent in masked hypertension than in white coat hypertension? Clin Exp Hypertens 2010; 32: 480-5.
6. Kawano Y, Horio T, Matayoshi T, Kamide K. Masked hypertension: subtypes and target organ damage. Clin Exp Hypertens 2008; 30: 289-96.

7. Drager LF, Diegues-Silva L, Diniz PM, et al. Obstructive sleep apnea, masked hypertension, and arterial stiffness in men. Am J Hypertens 2010; 23: 249-54.

8. Baguet JP, Lévy P, Barone-Rochette G, et al. Masked hypertension in obstructive sleep apnea syndrome. J Hypertens 2008; 26: 885-92

9. Quiñones MA, Otto CM, Stoddard M, Waggoner A, Zoghbi WA. Recommendations for quantification of Doppler echocardiography: a report from the Doppler Quantification Task Force of the Nomenclature and Standards Committee of the American Society of Echocardiography. J Am Soc Echocardiogr 2002; 15: 167-84.

10. Buysse DJ, Reynolds CF III, Monk TH, Berman SR, Kupfer DJ. The Pittsburgh Sleep Quality Index: a new instrument for psychiatric practice and research. Psychiatry Res 1989; 28: 193-213.

11. Pickering TG, Davidson K, Gerin W, Schwartz JE. Masked hypertension. Hypertension 2002; 40: 795-6.

12. Ohkubo T, Kikuya M, Metoki H, et al. Prognosis of "masked" hypertension and "white-coat" hypertension detected by 24-h ambulatory blood pressure monitoring 10-year follow-up from the Ohasama study. J Am Coll Cardiol 2005; 46: 508-15.

13. Angeli F, Reboldi G, Verdecchia P. Masked hypertension: evaluation, prognosis, and treatment. Am J Hypertens 2010; 23: 941-8.

14. Pierdomenico SD, Cuccurullo F. Prognostic value of white-coat and masked hypertension diagnosed by ambulatory monitoring in initially untreated subjects: an updated meta analysis. Am J Hypertens 2011; 24: 52-8.

15. Trevisol DJ, Moreira LB, Kerkhoff A, Fuchs SC, Fuchs FD. Health-related quality of life and hypertension: a systematic review and meta-analysis of observational studies. J Hypertens 2011; 29: 179-88.

16. Palagini L, Bruno RM, Gemignani A, Baglioni C, Ghiadoni L, Riemann D. Sleep loss and hypertension: a systematic review. Curr Pharm Des 2013; 19: 2409-19.

17. Erden I, Erden EC, Ozhan H, et al. Poor-quality sleep score is an independent predictor of nondipping hypertension. Blood Press Monit 2010; 15: 184-7.

18. Mansoor GA. Sleep actigraphy in hypertensive patients with the 'non-dipper' blood pressure profile. J Hum Hypertens 2002; 16: 237-42.

19. Hermida RC, Ayala DE, Mojón A, Fernández JR. Blunted sleep-time relative blood pressure decline increases cardiovascular risk independent of blood pressure level: the "normotensive non-dipper" paradox. Chronobiol Int 2013; 30: 87-98.

20. Wang C, Zhang J, Liu X, et al. Reversed dipper blood-pressure pattern is closely related to severe renal and cardiovascular damage in patients with chronic kidney disease. PLoS One 2013; 8: e55419.

21. Jennersjö $P E$, Wijkman $M$, Wiréhn $A B$, et al. Circadian blood pressure variation in patients with type 2 diabetes-relationship to macro- and microvascular subclinical organ damage. Prim Care Diabetes 2011; 5: 167-73.

22. Shimbo D, Newman JD, Schwartz JE. Masked hypertension and prehypertension: diagnostic overlap and interrelationships with left ventricular mass: the Masked Hypertension Study. Am J Hypertens 2012; 25: 664-71.

23. Kotsis V, Stabouli S, Toumanidis S, et al. Target organ damage in "white coat hypertension" and "masked hypertension". Am J Hypertens 2008; 21: 393-9. 
24. Mitsnefes M, Flynn J, Cohn S, et al.; CKiD Study Group. Masked hypertension associates with left ventricular hypertrophy in children with CKD. J Am Soc Nephrol 2010; 21: 137-44.

25. Oe Y, Shimbo D, Ishikawa J, et al. Alterations in diastolic function in masked hypertension: findings from the masked hypertension study. Am J Hypertens 2013; 26: 808-15. 